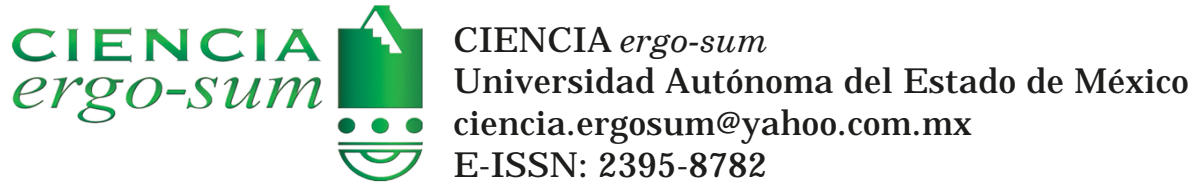

\title{
La neocogestión del conocimiento en la sociedad digital: una aproximación interpretativa
}

Rodríguez, Rosselys Carolina; Camejo, Armando

La neocogestión del conocimiento en la sociedad digital: una aproximación interpretativa

CIENCIA ergo-sum, vol. 27, núm. 1, marzo-junio 2020|e79

Universidad Autónoma del Estado de México, México

Esta obra está bajo una Licencia Creative Commons Atribución-NoComercial-SinDerivar 4.0 Internacional .

Rodríguez, R. C. y Camejo, A. (2020). La neocogestión del conocimiento en la sociedad digital : una aproximación interpretativa. CIENCIA ergo-sum, 27(1). https://doi.org/10.30878/ces.v27nla11 


\title{
La neocogestión del conocimiento en la sociedad digital: una aproximación interpretativa
}

The neo-co-management in the digital society: An interpretative approach

\author{
Rosselys Carolina Rodriguez \\ Universidad de Carabobo, Venezuela \\ rosselysr2@gmail.com \\ Armando Camejo \\ Politécnico Gran Colombiano, Colombia \\ armandocamejo@gmail.com
}

\author{
Recepción: 25 de abril de 2019
}

Aprobación: 20 de agosto de 2019

\section{RESUMEN}

Se realiza una aproximación interpretativa de la neocogestión del conocimiento en la sociedad digital y se da a conocer la dinámica actual del conocimiento en la vida de unos sujetos inmersos en un modelo social de base tecnológica. En relación con la metódica, se precisa como un estudio exegético de tipo documental. Adicionalmente, es esencial mencionar que durante el desarrollo del trabajo se encuentran hallazgos que evidencian el surgimiento de una nueva cogestión del conocimiento y permiten concluir que en ella se integran redes colaborativas entre los diversos actores (nodos) de la sociedad, quienes mediante las tecnologías de información y comunicación se apropian del conocimiento y lo desplazan al colectivo como un bien común.

Palabras claves: sociedad digital, conocimiento, apropiación, neocogestión, nodos.

\begin{abstract}
The article aims to do an interpretative approach of the neo-co-management knowledge in the digital society, letting know the current dynamics of knowledge in the lives of some subjects immersed in a technological-based social model. In relation to the method, it is definedas a documentary type descriptive study. Additionally, it is essential to mention that during the article development there were findings that show the emergence of a new co-management of knowledge and allow to conclude that in it, collaborative networks are integrated among the different actors (nodes) of society, who through technologies of information and communication, appropriate knowledge and displace it into the collective as a common well.
\end{abstract}

KEYWORDS: digital society, knowledge, appropriation, neo-co-management, nodes.

\section{INTRODUCCIÓN}

En la historia de la humanidad, la aparición de nuevas y mejores herramientas o tecnologías ha generado distintas posibilidades de ejecutar las tareas; hablamos de la entrada en vigencia de un nuevo paradigma que viene a modificar y en muchos casos a revolucionar la sociedad. Autores como Drucker (1969), Toffler (1979), Naisbitt (1982) y Senge (1990) fueron visionarios, pues anticiparon procesos de cambios que originarían una sociedad industrializada edificada sobre la tecnología. Este planteamiento, que en su momento era ficción, se materializó en la sociedad digital o sociedad de la información, modelo que ha despertado el interés de investigadores como Castells (2000) y Poe (2004), entre otros.

Esta nueva era social, cuyas infraestructuras están edificadas sobre las tecnologías de información y la comunicación, se caracteriza por el intercambio de datos en tiempo real de manera remota; en este sentido, al existir nuevas y mejores tecnologías, se genera una reacción en cadena que modifica los procesos inmersos en las formas de vida de los individuos: sus creencias, la moral, las costumbres, los hábitos, las habilidades y, en consecuencia, el conocimiento y sus formas de apropiación en la búsqueda del aprendizaje social. 
Con base en lo planteado, se establece como objetivo central de este artículo realizar una aproximación explicativa-interpretativa de la neocogestión del conocimiento en la sociedad digital. El texto se desarrolla con una metódica documental combinada con el uso del resumen analítico y el análisis crítico; todo orientado a interpretar la teoría existente sobre la sociedad digital, el conocimiento, la apropiación de éste y sus variables en función de construir los fundamentos de la neocogestión en este tipo nuevo arquetipo de sociedad.

Para alcanzar el objetivo trazado, se llevó a cabo una revisión exhaustiva de la bibliografía con relación a: a) navegamos realmente en una sociedad digital, $b$ ) se ha digitalizado el conocimiento, $c$ ) la apropiación del conocimiento ha permeabilizado la sociedad digital, $d$ ) el significado de la neocogestión del conocimiento en la era digital.

\section{Navegamos Realmente EN UNA SOCIEDAD Digital}

Para la década de los setenta, Alvin Toffler en su obra La tercera ola (1979: 155) predecía una sociedad con avances industriales y genéticos por la incorporación de tecnología, la desmasificación de los medios y una esfera de información derivada de una esfera tecnológica que guiaba la vida de los individuos. Ya se percibía la ruptura y fin de un paradigma, una revolución digital que desplegaba un modelo distinto de ideología con propuestas innovadoras que moldearon la estructura de la sociedad digital.

En la actualidad existe una permanente transformación social y cultural con rasgos claramente definidos dentro de los que destacan la dependencia tecnológica para el manejo de la información y la comunicación en todos los ámbitos, el fortalecimiento de la educación online, una economía alterna y su dinámica en el ciberespacio. En otras palabras, en este arquetipo social se acentúa la virtualización del ser, entendida como la proyección del hombre, los entes o las instituciones a la red, proceso mediante el cual se desvanecen y crean su identidad digital.

Desde esta perspectiva, la sociedad digital o de la información se complementa en una misma definición. Al respecto, Alfonso (2016: 236) precisa que "la sociedad de la información surge como un enfoque [...], que se caracteriza por un cambio de paradigma en las estructuras industriales y en las relaciones sociales”. En relación con ello, Castells (2000: 56) añade que:

Quisiera establecer una distinción analítica entre las nociones de 'sociedad de la información' y 'sociedad informacional', con implicaciones similares para la economía de la información/informacional. El término sociedad de la información destaca el papel de esta última en la sociedad. Pero yo sostengo que la información, en su sentido más amplio, es decir, como comunicación del conocimiento, ha sido fundamental en todas las sociedades [...]. En contraste, el término informacional indica el atributo de una forma específica de organización social en la que la generación, el procesamiento y la transmisión de la información se convierten en las fuentes fundamentales de productividad y poder, debido a las nuevas condiciones tecnológicas que surgen en este periodo histórico.

Castells argumenta que una sociedad en red es el espacio de los flujos (488):

El espacio de los flujos es la organización material de las prácticas sociales en tiempo compartido que funcionan a través de los flujos. Por flujo se entiende a las secuencias de intercambio e interacción determinadas, repetitivas y programables entre las posiciones físicamente inconexas que mantienen los actores sociales en las estructuras económicas, políticas y simbólicas de la sociedad. Las prácticas sociales dominantes son aquellas que están incorporadas a las estructuras sociales dominantes. Por estructuras dominantes entiendo los dispositivos de organizaciones e instituciones cuya lógica interna desempeña un papel estratégico para dar forma a las prácticas sociales y la conciencia social de la sociedad en general.

De lo anterior se deduce que la sociedad digital o de la información es aquella donde fluye la información permanentemente sin barreras de tiempo y espacio entre los distintos actores; la tecnología está inmersa en todas las 
actividades cotidianas de los individuos definiendo los modos de vida. Sin embargo, la sociedad de cada país será digital en mayor o menor proporción, según sus condiciones, tomando como referencia el grado de desarrollado, dado que se requiere para su dinámica una infraestructura tecnológica y energética, lo cual aumenta o disminuye la brecha digital existente entre las naciones.

Una vez conceptualizada la sociedad digital, desde la postura de Delarbre (2001: 1) se describen algunas de sus características:

- Exhuberación: volumen inacabable de información.

- Omnipresencia: los nuevos instrumentos de información se encuentran por doquier, forman parte del escenario público contemporáneo.

- Irradiación: la información se distingue por la distancia hoy prácticamente ilimitada que alcanza el intercambio de mensajes.

- Velocidad: la comunicación, salvo fallas técnicas, se ha vuelto instantánea.

- Multilateralidad/centralidad: las capacidades técnicas de la comunicación contemporánea permiten que se reciba información de todas partes en tiempo real.

- Interactividad/unilateralidad: los nuevos instrumentos para propagar información permiten que sus usuarios sean consumidores y productores de mensajes al mismo tiempo.

- Desigualdad: el internet continúa siendo ajeno para mucha gente sobre todo en los países más pobres o incluso en zonas o entre segmentos de la población marginada aún en los países más desarrollados.

- Heterogeneidad: en los medios contemporáneos, y particularmente en el internet, se duplican y multiplican actitudes, opiniones, pensamientos y circunstancias que están presentes en nuestras sociedades.

- Desorientación: la enorme y creciente cantidad de información se ha convertido en desafío cotidiano y en motivo de agobio para quienes reciben o pueden encontrar millares de noticias, símbolos, declaraciones, imágenes e incitaciones de casi cualquier índole a través de los medios y especialmente en la red de redes.

En esa misma dirección, Castells (2014) realiza una compilación de datos que demuestran la veracidad de las diferencias indicadas (tabla 1).

TABLA 1

Impacto de internet en la sociedad

\begin{tabular}{|cc}
\hline 1996 & Usuarios de internet \\
& 40 millones \\
2013 & 2500 millones (la mayoría en China) \\
& Suscriptores de comunicaciones inalámbricas \\
2091 & 16 millones de suscriptores con dispositivos inalámbricos \\
& 7000 millones de suscriptores con dispositivos inalámbricos (so- \\
& bre una población mundial de 7700 millones de habitantes) \\
& Usuarios de las redes sociales \\
& 1000 millones de usuarios \\
2010 & 2000 millones de usuarios aproximadamente \\
\hline Porcentaje de información digitalizada para 2014 (de toda la información existente) & Información digitalizada y accesible en internet y otras redes \\
$95 \%$ & informáticas \\
\hline
\end{tabular}

Fuente: elaboración propia a partir de Castells (2014). 
Vale destacar que las características puntualizadas vistas en conjunto con los datos que anteceden permiten afirmar que esta es una sociedad digital donde las formas de vidas de los individuos se desarrollan mediante el uso de tecnología, excepto las zonas marginadas del mundo. A continuación, se profundizará sobre las repercusiones que genera esta sociedad en el proceso de transformación de la información en una base cognitiva esencial para la resolución de problemas colectivo.

\section{Se ha digitalizado el CONOCimiento}

Desde tiempos arcaicos el hombre ha tenido una necesidad de buscar la verdad de todo. Durante este proceso de investigación han cambiado los modelos sociales y por consiguiente sus características convirtiendo inconmensurable la tarea de generar conocimiento, motivo por el cual se ha obligado a la sociedad a reevaluar su forma de construcción en la actualidad.

Al referirse al conocimiento, Ramírez (2009: 217) explica que es el acto consciente e intencional para aprehender las cualidades del objeto y es referido primariamente al sujeto. Al respecto, Luhmann (1998: 166) indica que:

El conocimiento es un esquema cognitivo que se considera verdadero, pero que, al mismo tiempo, es variable. El conocimiento no representa el mundo de forma objetiva, pero hay un criterio para su adecuación (su verdad) que reside en su convalidación en la práctica aunque estos efectos prácticos no están dados de forma objetiva, sino que a su vez se constituyen a través de las interrelaciones entre las personas perceptoras y actuantes por un lado y la realidad por otro lado. De esta forma, se construye socialmente una certeza de la realidad que es condición imprescindible para cualquier formar de pensar y de actuar.

Como ya se explicó, en cada época se han aportado elementos al proceso de construcción del conocimiento. La figura 1 muestra el proceso.

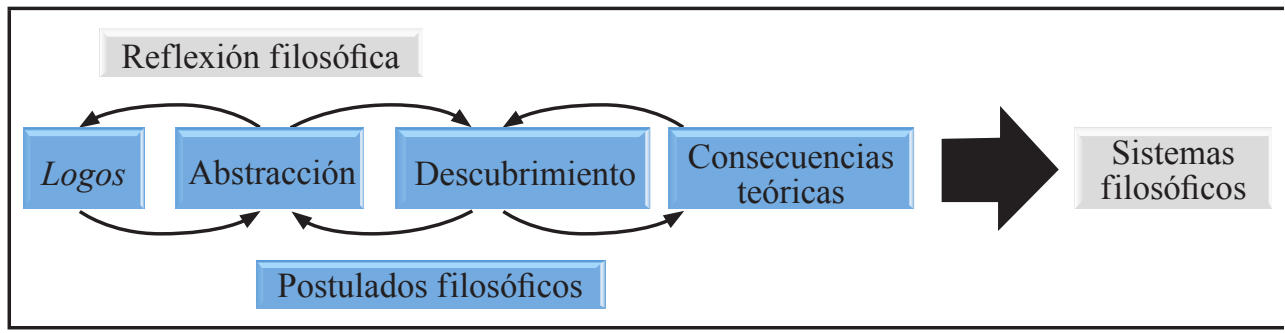

FIGURA 1

La construcción* del conocimiento científico en las ciencias sociales

Fuente: Zambrano (2017).

Nota: *Construcción desde la filosofía de la ciencia.

De acuerdo con la figura 1, durante la construcción del conocimiento que genera el comportamiento en la sociedad se trabajan tres dimensiones: una ontológica, una epistemológica y otra metodológica, cada una con su área de acción definida; la primera busca entender la composición de la realidad y las entidades que forman parte de ella, la segunda se relaciona con las teorías que explican cómo se aborda esta realidad, en otras palabras, incluye todo un herramental teórico para conocerla y, por último, la metodológica envuelve los medios con los cuales se capturan los elementos sustanciales del contexto donde se da el problema estudiado.

En este orden de ideas, la abstracción del fenómeno a estudiar se puede hacer por inducción, deducción o abducción. De acuerdo con Osorio (2007: 153), "la inducción es un procedimiento del razonamiento que va de lo particular a lo general. Se parte de hechos particulares (casos concretos) y se llega a conclusiones generales acerca del tipo al que pertenecen los hechos particulares considerados". En la deducción contrariamente se parte de los hechos generales a los particulares. La abducción comprende, por su parte, extraer conjeturas del descubrimiento, contrastarlas y discutirlas. 
Otro aspecto importante a considerar es que la selección del paradigma epistemológico afecta cómo se abordará la investigación. Desde la perspectiva de Carr y Kemis (1988) pueden reconocerse tres paradigmas, como se evidencia en cuadro sinóptico de la figura 2.

\begin{tabular}{|l} 
1. El positivista \\
$\qquad \begin{array}{l}\text { 1.1. Denominado: empírico, analítico, objetivista, realista, cuanti- } \\
\text { tativo y racionalista } \\
\text { 1.2. Relación sujeto-objeto: independiente, investigador externo } \\
\text { 1.3. Objetivos: describir, analizar, explicar, predecir, controlar, } \\
\text { verificar o construir teorías o leyes } \\
\text { 1.4. Fundamentos teóricos: el positivismo y el empirismo } \\
\text { 1.5. Lógica: hipotética-deductiva (la abducción forma parte de } \\
\text { este método) e inductiva }\end{array}$ \\
$\qquad \begin{array}{l}\text { 2.1. Denominado: humanista, hermenéutico, cualitativo, etnográfico, } \\
\text { fenomenológico y constructivista } \\
\text { 2.2. Relación sujeto-objeto: dependiente, existe interrelación } \\
\text { 2.3. Objetivos: comprender, interpretar y descubrir } \\
\text { 2.4. Fundamentos teóricos: la fenomenológica, la teoría interpretativa } \\
\text { 2.5. Lógica: inductiva, descriptiva e intersubjetiva }\end{array}$ \\
$\left\{\begin{array}{l}\text { 3.1. Denominado: socio crítico, cambio, acción y racionalidad } \\
\text { 3.2. Relación sujeto-objeto: se basa en la interrelación } \\
\text { 3.3. Objetivos: liberar, emancipar, transformar, criticar y generar } \\
\text { potencial de cambio } \\
\text { 3.4. Fundamentos teóricos: la teoría crítica } \\
\text { 3.5. Lógica: inductiva }\end{array}\right.$ \\
\hline
\end{tabular}

FIGURA 2

Aspectos a considerar en la selección del paradigma epistemológico Fuente: elaboración propia a partir de Carr y Kemis (1988).

Como se puede visualizar, ya existe una gama de conocimientos generados en los distintos modelos de sociedad, así como instrumentos de rigor científico que se utilizan sistemáticamente para llevar a cabo el proceso de investigación y por ende la generación de conocimiento. En tal sentido, se puede dilucidar que el conocimiento en la actualidad es la acción de comprender hechos relacionados con el Ser en función del criterio de verdad, en este tiempo construido sobre la interpretación del lenguaje, las tecnologías e interacciones de los actores en la red que estructuran la forma actual de ver el mundo.

No obstante, la tecnología produce un volumen ilimitado de información proporcionando un cúmulo disponible para la compresión teorética de los fenómenos. En este sentido, se requiere la alineación entre la apropiación del conocimiento, la epistemología como fuente de estudio y la tecnología como herramienta para filtrar la información relevante en pro de la construcción cognitiva bajo el criterio de verdad que rige el colectivo.

Es indispensable para ello la incorporación de herramientas de análisis tanto cualitativas como cuantitativas para filtrar la información fiable y oportuna utilizada en el proceso de investigación y por consiguiente en el desarrollo del conocimiento, como lo son los software; entre los más destacados se encuentran el Atlas.ti y el programa estadístico SPSS, Stella, SocNetV (Social Networks Visualizer), GraphML, GraphViz, Adyacencia, Pajek, UCINET, GEPHY, BigPicture, DecisionTools Suite, SAS, BMDP, SYSTAT, STATISTICA, STATA, MINITAB, S-PLUS, EVIEWS, STAT-GRAPHICS y MATLAB, R, Past, Open Stat, GenStat Discovery Edition, QDA Miner Ethnograph, Nvivo, MaxQDA, BigPicture y DecisionTools, al igual los gestores bibliográficos como Zotero y Mendeley y por último, pero no menos importante, el software de planificación Project. 
De los anteriores planteamientos se deduce que la producción de conocimiento y el conocimiento en sí se ha estado digitalizando en la medida en que se le han ido incorporando específicos tipos de software a cada una de las actividades de los procesos de investigación, ya que están permitiendo captar las huellas digitales dejadas en las redes, transformarlas en datos digitales, y, por medio de dichos softwares, procesarlos en tiempo real. De este modo, las sociedades del conocimiento, por medio de la TIC, han estado digitalizando, como ya se ha dicho, tanto el proceso de producción de conocimiento como al conocimiento mismo.

\section{LA APROPIACIÓN DEL CONOCIMIENTO HA PERMEABILIZADO LA SOCIEDAD DIGITAL}

Según Chaparro (1998: 10), un "proceso de aprendizaje social” se logra cuando el conocimiento individual y vivencial se codifica y se logra socializar en una comunidad o una empresa desarrollando capacidades y habilidades en las personas y en las organizaciones que les permite responder con éxito a cambios permanentes en su entorno, así como a los desafíos y oportunidades que este entorno les brinda.

El término apropiación del conocimiento según Domínguez (2016) se puede evaluar desde tres perspectivas:

a) Como consigna: expresa que toda sociedad debe entender en qué consisten y cómo se crean los conocimientos, los cuales son de dominio público.

b) Como derecho: todos los actores sociales que estén en condiciones de hacerlo pueden participar en la creación de conocimientos; por tal motivo, las políticas en esta materia deben establecer las circunstancias para que se acceda a los centros de producción de conocimientos en todos los niveles.

c) Como práctica: la sociedad necesita transformar sus modos cotidianos de existencia para obtener seguridad. Al respecto, el sistema educativo y el sistema de medios de comunicación han de garantizar el libre intercambio de nuevos conocimientos y de opiniones acerca de su impacto en lo cotidiano y en lo trascendente de cada persona, familia, región o nación.

De lo que trata la apropiación social del conocimiento, es que los individuos participen activamente en la producción, adaptación, consumo, aplicación de conocimientos, con el fin de acceder a sus beneficios de manera que mediante sus actuaciones estos sujetos permitan que el conocimiento mute y se acople a los rasgos distintivos del modelo social vigente, los cuales están sometidos a su vez a un entorno convulsionado que los obliga a adaptarse a sus modificaciones, en otros palabras, se evidencia un proceso en red donde cada uno de los elementos inciden en el otro dando cabida a una nueva matriz cognitiva y por ende un cambio social.

Por esta vinculación entre los diversos actores de la sociedad, se considera pertinente hacer referencia a una de las teorías que trata de ayudar a explicar los procesos de producción, adaptación, consumo y aplicación de conocimiento: el conectivismo de Siemens (2006). Esta teoría promueve un modelo de aprendizaje como actividad colectiva. Estos tipos de procesos, como refiere el autor, son en redes (Siemens, 2006: 29):

El acto de aprender es un acto de creación de una red externa de nodos, donde conectamos y damos forma a fuentes de información y de conocimiento. El aprendizaje ocurre en nuestras cabezas es una red interna (neurálgica). Las redes de aprendizaje pueden ser percibidas entonces como estructuras que creamos con el fin de mantenernos al día y continuamente adquirir, experimentar, crear y conectar nuevos conocimientos (externos).

De la narrativa previa se desprende que los procesos de producción, adaptación, consumo y aplicación del conocimiento, se logran estableciendo redes de aprendizajes que incluyen nodos representados por los entes actuantes en la sociedad desde una dimensión virtual. Estas redes contienen subestructuras internas, vistas como arquetipos cognitivos para el entendimiento de los flujos. Los entes están representados no por entidades individuales sino colectivas como la familia, las organizaciones, el Estado y las universidades, cuyas preferencias se orientan la red; un ejemplo son el teletrabajo, procesos de formación E-learnig y el comercio electrónico. 
Sin embargo, a pesar de todos los avances en tema de apropiación del conocimiento, este proceso no ha logrado adherirse a las distintas aristas de la sociedad y sólo puede identificarse en instituciones educativas dentro de las que destacan las universidades, razón por la cual se hace imperativo, por un lado, establecer conexiones entre éstas y el resto de los nodos (el Estado, las organizaciones y la familia), en función de posicionar el conocimiento al alcance de todos y, en segundo, proporcionar un herramental tecnológico, así como acceso a internet a quienes estén marginados en este tipo de sociedad.

\section{ANÁLISIS PROSPECTIVO: EL SIGNIFICADO DE LA NEOCOGESTIÓN DEL CONOCIMIENTO EN LA ERA DIGITAL}

Cuando se habla de la sociedad red o de la información, se tiende a una estructura social con una cultura de participación virtual y una construcción de conocimiento donde los nodos pasan a ser sujetos activos productores de información, interacciones y nuevos significados. Por las características peculiares de este nuevo orden social postmoderno, se alude a la teoría de la conectividad, que encaja perfectamente en su dinámica y se incorpora como elemento de la neocogestión del conocimiento, expresión que se desarrollará en los próximos párrafos y abarcará los nodos, la conectividad y el flujo de información.

La expresión neocogestión, incluye el prefijo neo cuyo significado, de acuerdo con el Diccionario de la Real Academia Española (2018), significa: nuevo, reciente; por otra parte, Pérez Martelo Vinck (2009: 134), define cogestión como:

Un proceso de conformación de arreglos sociotécnicos, en el cual no hay una relación lineal productor-usuario, ya que estos roles son intercambiables en el tiempo, y están mediados por sucesivas traducciones. Los aprendizajes atraviesan las instituciones y disciplinas y se estructuran diferentes esquemas de trabajo entre áreas, con una negociación de roles y tiempos de desarrollo.

Adicionalmente, Delgado Bello (2014: 575), define la cogestión como: "Una alianza entre partes con identidades distintas que deciden llevar juntas algún emprendimiento”.

Para complementar el alegato que antecede, y parafraseando a Brynjolfsson y McAfee (2013), se indica que los modos de producción en la segunda era de las máquinas se erigen sobre cuatro factores básicos: la propiedad intelectual, el capital organizacional, el talento humano y la generación de contenidos por parte de usuarios, lo que implica conexiones entre las partes para el logro de los objetivos colectivos.

De lo explicado, se arguye que la neocogestión implicará arreglos sociotécnicos o alianzas entre partes, identificadas en este caso como nodos que se integran a redes, los cuales deben conectarse, generar y modelar información que será fuente de conocimiento.

Dichos nodos, en este caso identificados como la familia, el Estado, las organizaciones y las universidades se integrarán a redes colaborativas participando activamente en la producción, adaptación, consumo y aplicación de conocimientos. Todos los cuales, mediante las tecnologías de información, accederán a los beneficios de la comprensión social y la convertirán en modelo para el discernimiento en las actividades que desempeñen, en función de alcanzar el conocimiento como un bien común que se traduzca en una mejora de la calidad de vida y en un bienestar social global.

El conectivismo regirá el flujo de información en distintas direcciones, donde cada nodo debe salir de su zona de confort e involucrarse en el manejo de herramientas tecnológicas con el fin de superar las barreras generadas por el gran volumen de información y como responsable del logro de los objetivos de la red. La complementariedad, el apoyo mutuo y la sinergia serán rasgos determinantes para el éxito del proceso de apropiación de conocimiento mediante la neocogestión.

Dentro de las conexiones principales que deben existir en una sociedad, extraídas del flujo de información entre los nodos, están: 
a) Primera conexión: entre familia y organizaciones, cuyo objetivo será desarrollar habilidades tecnológicas para la vida y el trabajo.

b) Segunda conexión: entre organizaciones y universidades, orientada a fomentar competencias y conocimientos digitales, para la cual deberán realizarse las adaptaciones curriculares necesarias para generar los tipos de profesionales que requiere la era digital.

c) Tercera conexión: entre universidades y Estado, enfocada a desarrollar la comunicación y colaboración para la construcción de políticas apropiadas al contexto de la sociedad digital, así como políticas orientadas a la minimización de la brecha digital existente.

d) Cuarta conexión: entre Estado y familia, dirigida a fomentar políticas para el desarrollo de una ética y cultura de innovación en la solución de problemas. Esta dinámica se explica a continuación en la figura 3.

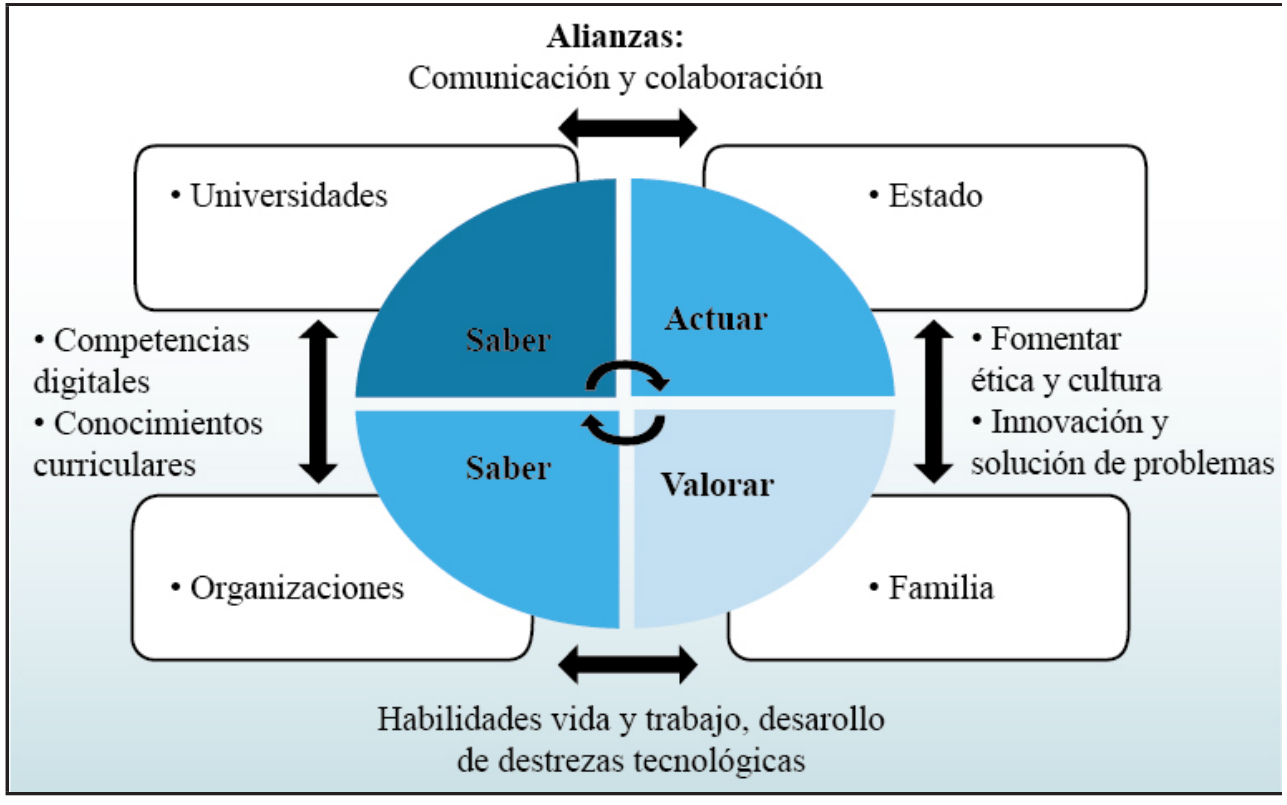

FIGURA 3

Neocongestión del conocimiento en la sociedad digital

Fuente: elaboración propia.

Todo este cambio de paradigma e integración de redes y subestructuras para la solución de problemas sociales buscaría, de acuerdo con López (2011:127), la construcción de una filosofía que abra el espacio a una nueva epistemología centrada en la red y sus conexiones y en la interpretación como función privilegiada en el saber.

\section{Conclusiones}

El nuevo modelo de verdad de la sociedad digital sobre la que se ha argüido en los apartados anteriores se caracteriza por la comunicación y las tecnologías. Cabe decir que dichas palabras regirán los sistemas que definirán los modos de vidas de los individuos de esta era.

Las conclusiones a las que se han llegado se encuadran en los siguientes aspectos:

1. En la actualidad se navega en una sociedad digital, donde existe una dinámica social y cultural en permanente cambio, una dependencia tecnológica para el manejo de la información y la comunicación en todos los ámbitos, el fortalecimiento de la educación online, una economía alterna que funciona en el 
ciberespacio. En otras palabras, los modos de vidas de los individuos se desarrollan mediante el uso de tecnología.

2. No sólo los modos de vida han mutado hacia la digitalización, también el conocimiento lo ha hecho, se ha digitalizado. Y ha sido así porque, a pesar de que en el siglo XX ya había comenzado a variar la noción ontológica con Heidegger y el giro lingüístico de la filosofía, la digitalización acelerada, producida por la incorporación a la metodología, diseño de software y APP para cada fase de la investigación, han forzado estos cambios en la ontología y la epistemología. De modo que las tres dimensiones de la construcción del conocimiento: la ontológica, la epistemológica y la metodológica han variado al tenor de la digitalización que caracteriza a las sociedades red.

3. Se evidencian diferentes grados de progresos del tema de apropiación del conocimiento. Primero: se encuentran países desarrollados que han logrado integrar eficientemente la generación de conocimiento en todas sus aristas. Segundo: países en vía de desarrollo que no han logrado que este proceso de generación de conocimiento se infiltre en todos los nodos de la sociedad, y el cual esté limitado a las instituciones educativas dentro de las que destacan las universidades. Tercero: regiones que están al margen de la tecnología que aún mantiene un proceso primitivo. Para estos últimos dos casos se hace obligatorio fomentar conexiones entre todos los elementos de la sociedad en función de posicionar el conocimiento al alcance de todos y, a su vez, proporcionar un herramental tecnológico y acceso a internet a quienes estén marginados en este tipo de sociedad para evitar la exclusión.

4. Para finalizar es indispensable impulsar la neocogestión y los arreglos sociotécnicos o alianzas entre los nodos que integran la red sociedad, los cuales deben conectarse, generar y modelar la información que será fuente del conocimiento adaptado a esta modelo de sociedad.

\section{Agradecimiento}

Se agradece la revisión crítica al contenido y los aportes sustanciales realizados por los árbitros y el comité editorial de la revista.

\section{Referencias}

Alfonso, I. (2016). La sociedad de la información, sociedad del conocimiento y sociedad del aprendizaje. Referentes en torno a su formación. Bibliotecas. Anales de investigación, 12(2), 235-243.

Brynjolfsson, E. y Mcafee, A. (2013). The second machine age. W.W. Norton Company.

Carr, W. y Kemmis, S. (1988). Teoría critica de la enseñanza. La investigación-acción en la formación del profesorado. Barcelona: Martínez Roca.

Castells, M. (2014). El impacto de internet en la sociedad: una perspectiva global. Disponible en https://www. bbvaopenmind.com/articulos/el-impacto-de-internet-en-la-sociedad-una-perspectiva-global/.

Castells, M. (2000). La era de la información: economía, sociedad y cultura. Volumen I: La sociedad red. Madrid: Alianza Editorial.

Chaparro, F. (1998). Apropiación social del conocimiento, aprendizaje y capital social. Simposio sobre Biotecnología y Bioseguridad. Washington: Banco Mundial. 
Delarbre, R. T. (2001). Vivir en la sociedad de la información: orden global y dimensiones locales en el universo digital. Revista Iberoamericana de Ciencia, Tecnologia, Sociedad e Innovación, 1. Disponible en https:// dialnet.unirioja.es/servlet/articulo?codigo $=741540$.

Delgado Bello, L. A. (2014). Innovación en gestión en Venezuela: trabajadores asociados y empresarios cogestionan una marca transnacional. Disponible en https://www.sommetinter.coop/sites/default/files/article cientifique/files/2014_37_delgado.pdf

Diccionario de Real Academia Española (2018). Disponible en http://www.rae.es/

Drucker, P. (1969). The age of discontinuity: Guidelines to our changing society. New York: Harper \& Row.

Domínguez, E. (2016). Apropiación del conocimiento. Foro del Oriente, Diálogo de saberes y oportunidades de región. Universidad de Antioquia. Disponible en www.udea.edu.co/wps/wcm/.../udea/.../Apropiación+social+del+conocimiento..pptx

López, F. (2011). Los extravios de la academia y la reforma de los bárbaros. Valencia: APUC, Asociación de Profesores, Universidad de Carabobo.

Luhmann, N. (1998). Sistemas sociales. Lineamientos para una teoría general. Barcelona: Anthropos.

Naisbitt, J. (1982). Megatrends: Diez nuevas Direcciones. Transformar nuestras vidas. Warner Books.

Osorio, F. (2007). Epistemología de las ciencias sociales: breve manual. Chile: Ediciones UCSH.

Poe, R. (2004). Ola 3: la nueva era en network marketing. Time \& Money Network Editions.

Pérez Martelo, C., y Vinck, D. (2009). Redes socio técnicas de cogestión de conocimiento en nanotecnologías en Colombia: ¿entre la visibilidad internacional y la apropiación local? Redes, 15(29), 113-137.

Ramírez, A. (2009). La teoría del conocimiento en investigación científica: una visión actual. Anales de la Facultad de Medicina, 70(3), 217-224. Disponible en <https://revistasinvestigacion.unmsm.edu.pe/index. php/anales/article/view/943>. [Consulta el 30 de agosto del 2018]

Senge, P. (1990). La quinta disciplina. El arte y la práctica de la organización abierta al aprendizaje. España: Granica. Siemens, G. (2006). Conociendo el conocimiento. Disponible en http://www.nodosele.com/editorial

Toffler, A. (1979). La tercera ola. Editorial Digital Lestrobe.

Zambrano, C. (2017). Métodos y metodologías aplicadas a la investigación en ciencias económicas y sociales. Venezuela: Universidad de Carabobo.

\section{BY-NC-ND}

